\title{
Editorial
}

\section{Peri-Venous Counter Spread — Be Prepared}

\author{
Howard S. Smith, MD', Gabor B. Racz, MD², and James E. Heavner, DVM, PhD²
}

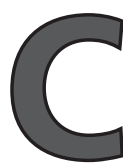

ervical interventional chronic pain therapies generally involve injecting small volumes of fluid into the cervical epidural space via transforminal or interlaminar routes. Subsequent rapid fluid spread in the ventrolateral space may lead to local increases in epidural pressure. Fortunately, the fluid normally spreads laterally and the pressure returns to normal.

The following actual case illustrates one potential pitfall of cervical interventional procedures. A 37-yearold right-handed white female complained of severe headaches and upper neck pain on the right side present for many years. She had been seen by numerous physicians and received many treatments all of which were ineffective. She was eventually diagnosed with occipital neuralgia. Occipital nerve blocks administered multiple times produced good results. A neurosurgeon transected the occipital nerve and the patient's pain improved initially, but in 4 to 6 months the pain returned. After about 4 more months, the frequency of her headaches increased from 4 times a month to about 6 times a month.

Subsequently, the patient was diagnosed with cervical radiculopathy and a cervical epidural adhesiolysis was performed. The epidural catheter was threaded to the right side of the neck past the C-5-6 area where an epidurogram revealed a filling defect indicating epidural scaring was present. Stimulation of the right upper cervical nerve roots reproduced the patient's right-sided neck pain, supporting the clinical impression that these neural structures contributed to generation of the neck pain. The patient was sedated with a total of $50 \mathrm{mg}$ of meperidine and $5 \mathrm{mg}$ of diazepam and was responsive to verbal stimuli. Injection of local anesthetic did not produce motor block, indicating that there was no partial subdural or subarachnoid injection. Contrast was injected, followed by hyaluronidase, local anesthetics, and steroids. In the recovery area, the injection produced pain affecting the right side of the neck. The patient described the pain as radiating from the face to the ear, neck, right upper extremity, and subsequently all the way down to the right leg. The patient described pain affecting the right upper extremity - progressing to numbness and inability to move the right side. Additional sedation was given and the patient discharged home. Three days later a CT scan showed no evidence of spinal cord injury or hematoma formation. Neurological and neurosurgical consultation was sought. Right sided weakness persisted.

Five days later an MRI study revealed cord edema from $\mathrm{C} 2$ to $\mathrm{C} 7$.

Lower extremity function recovered essentially to normal in $4-5$ months. Two years later, upper extremity function had improved significantly and, following appropriate physical therapy, had returned to the point where the patient was able to resume writing with the right hand. Ten years later, some residual weakness in pronation was still present. The most likely cause of the course of events following the cervical injection was compromise of spinal cord blood supply.

From: ${ }^{1}$ Albany Medical College, Albany, NY; ${ }^{2}$ Texas Tech University, Texas Tech University Health Sciences Center, Lubbock, Texas.

Dr. Smith is Editor-in-Chief of Pain Physician, Associate Professor \& Academic Director of Pain Management, Albany Medical College, Albany, New York.

\section{E-mail:smithh@mail.amc.edu}

Dr. Racz is Professor and Chairman Emeritus, Texas Tech University Health Sciences Center, Lubbock, Texas. Dr. Heavner is a Professor, Texas Tech University Health Sciences Center, Lubbock, Texas.

Disclaimer: There was no external funding in the preparation of this manuscript. Conflict of interest: None. 
The blood which nourishes the spinal cord is supplied by one anterior and 2 posterolateral spinal arteries which extend longitudinally in a variable fashion. The anterior spinal artery (ASA) supplies roughly $75 \%$ of the spinal cord, including the anterior horn motor neurons. At many levels, the spinal arteries receive blood from the radicular arteries, which enter the canal along with the nerve roots. The anterior spinal artery gives rise to the central arteries, each of which enters the spinal cord to supply the anterior horn and the anterior part of the lateral column on the left or right side at each level. The spinal arteries are connected by a pial plexus that surrounds the spinal cord, and the posterior spinal arteries may be linked together $(1,2)$. Each radicular artery supplies a separate functional region of the spinal arteries, particularly the anterior spinal artery (3). The first region extends from C1 until T3 and is supplied at the C3 level from the vertebral arteries (4) and at the level from C6 until C7 from the cervical ascending arteries $(1,4)$. The second region extends from T3 until T7 and sometimes receives a branch from the intercostals artery at the T7 level (5). The third region extends from $\mathrm{T} 8$ to the cone and receives a branch - arteria radicularis magna (ARM) (Adamkiewicz artery) from the intercostal artery, most frequently between T9 and T12 (6). There is sometimes a cone artery originating from the internal iliac artery (Desproges-Gotteron artery) at the L2 or L5 level (7-9).

de Haan et al (10) used a porcine model investigated whether spinal cord blood supply becomes dependent on other, noncritical, segmental arteries if spinal cord perfusion pressure (SCPP) is decreased $(11,12)$. The SCPP is equal (to the mean arterial pressure [MAP] minus the central venous pressure [CVP]) minus the cerebrospinal fluid (CSF) pressure (SCPP $=$ [MAP-CVP] - CSF) (10). Thus, SCPP may be reduced secondary to decreasing MAP and/or increasing CSF pressure. Similar to autoregulation of cerebral blood flow, autoregulation of spinal cord blood flow (measured by the hydrogen clearance technique) above a SCPP of $50 \mathrm{~mm} \mathrm{Hg}$ was observed (13). Below perfusion pressures of $50 \mathrm{~mm} \mathrm{Hg}$, SCBF decreased in proportion with decreasing perfusion pressure (13).

Adequacy of spinal cord perfusion may be assessed with transcranial electrical stimulation (tc-MEPs) as this technique is sensitive to changes in the functional integrity of the ischemia-sensitive motoneuronal pathways, including the anterior horn motor neurons, largely located in the anterior two-thirds of the spinal cord and are supplied by the ASA (de10). Tc-MEPs selectively reflect transmission in spinal cord motoneuronal pathways and can detect ischemia within minutes after the interruption of spinal cord blood supply (14). In humans undergoing TAA surgery, an acute interruption of spinal cord blood supply results in a rapid decrease of tcMEP amplitude to values below $25 \%$ of baseline without significant latency prolongation (14). A reduction of tc-MEP amplitude of one or both quadriceps muscles to less than $25 \%$ of baseline was considered an indication of ischemic spinal cord dysfunction.

Before noncritical segmental arteries were clamped, ischemic tc-MEP changes occurred when the SCPP was below $15 \pm 5$ (SD) $\mathrm{mm} \mathrm{Hg}$ (10). With a total of $9 \pm 3(S D)$ segmental arteries clamped, the ischemic SCPP threshold was $48 \pm 14 \mathrm{~mm} \mathrm{Hg}(P<0.01)$. After the release of all clamps, ischemia occurred at a SCPP of 15 \pm 5 (SD) $\mathrm{mm} \mathrm{Hg}$ (10). The usual SCPP ischemic threshold is roughly $50 \mathrm{~mm} \mathrm{Hg}$, however it may be difficult to predict a precise ischemic SCPP threshold for any particular individual patient. Multiple factors may affect an individual patient. Multiple factors may affect an individual's SCPP ischemic threshold including local CSF pressure, MAP, presence of stenotic lesions, diminished compliance, or decreased vasodilatory reserve in the critical or noncritical (which in certain circumstances may become important) blood vessels supplying the spinal cord.

If spinal cord compression leads to spinal cord ischemia and then is relieved quickly enough, there is a very good chance of full or almost full reversible recovery from ischemic insult/neurological dysfunction. In studies conducted by Shchurova and Khudiaev (15), inspection of the spinal cord after its open decompression showed an increase in capillary blood flow by $117.1 \pm 12.0 \%$ (from 50 to $300 \%$ ) in the spinal cord, its membranes, and radices in the compression zone, and by $10-50 \%$ in adjacent regions in $50 \%$ of cases. During the postoperative period, these patients exhibited marked improvement of neurological symptoms. Leg muscle strength increased by $175.3 \pm 3.6 \%$. The degree of the abnormalities in temperature and pain sensitivities went down by 3 to 5 dermatomes. The pain threshold decreased by $1.9 \pm 0.2^{\circ}$. Bowel or bladder functions improved significantly or werecompletely restored (15).

Patients whose blood flow in the injury focus and adjacent regions increased by only $5-30 \%(8.6 \pm 3.1 \%)$, after the decompression procedures did not show considerable improvement. The increase in their leg muscle strength was lower $(59.4 \pm 12.5 \%)$, there was no significant change in level of functional disturbance and the 
pain threshold changed nonsignificantly, and bowel/ bladder function was only slightly improved (15).

It appears that for the most part, epidural veins are valveless and devoid of classic bicuspid valves (16). Thus, there is no fixed anatomic barrier completely preventing reflux. However, there are at least 4 antireflux mechanisms that may contribute to effectively being an incomplete functional barrier to reflux (17). Four structures were encountered that might serve as an anti-reflux-mechanism: 1) intravenous dural folds, 2) meandrous configuration, 3) narrowing of the radicular veins at the point of penetration of the dura mater, and 4) varying numbers of smooth muscle fibers in the walls of the intradural and extradural parts of the radicular veins (17).

Furthermore, extremely dilated and tortuous vessels in the epidural cavity may also serve as a functional barrier to flow from the epidural to the subarachnoid space. The size differences, especially with the narrowed dural penetrating vein, leads to redirection of venous flow from the epidural veins towards the outside of the spinal canal to the inferior vena cava. In the clinical arena epidural intravenous injection of contrast is always observed to spread in the above described direction. Additionally when intravenous injection occurs during transforaminal or intralaminar injections the observed flow is always away from the spinal cord, to the outside of the spinal canal towards the vena cava (Fig. 1 ).

Evidence exists that "compartments" may be present in the epidural cavity, and when fluid is injected it sequentially fills these compartments as the pressure in each compartment exceeds the pressure needed to break tissue barriers to intercompartmental flow in order to allow spread of fluid into the adjoining compartment (18).

The capacitance of the epidural cavity may be reduced with restricted outflow when the space is potentially limited by epidural scar formation, fibrosis, disk prolapse, trauma, surgery, or age-related changes. Fluid follows the path of least resistance and may initially flow into the epidural compartment with diminished compliance and capacitance and then become "trapped" with a steep rise in pressure. If the fluid is unable to "escape" or "runoff laterally" then this loculation of fluid may quickly build up an intense pressure. This high pressure may compromise blood supply to the spinal cord with potential resultant ischemic and/or traumatic insult.

A recently described additional mechanism for compromise of spinal cord blood supply is opening of $\mathrm{A}-\mathrm{V}$ fistulas producing increasing venous pressure and

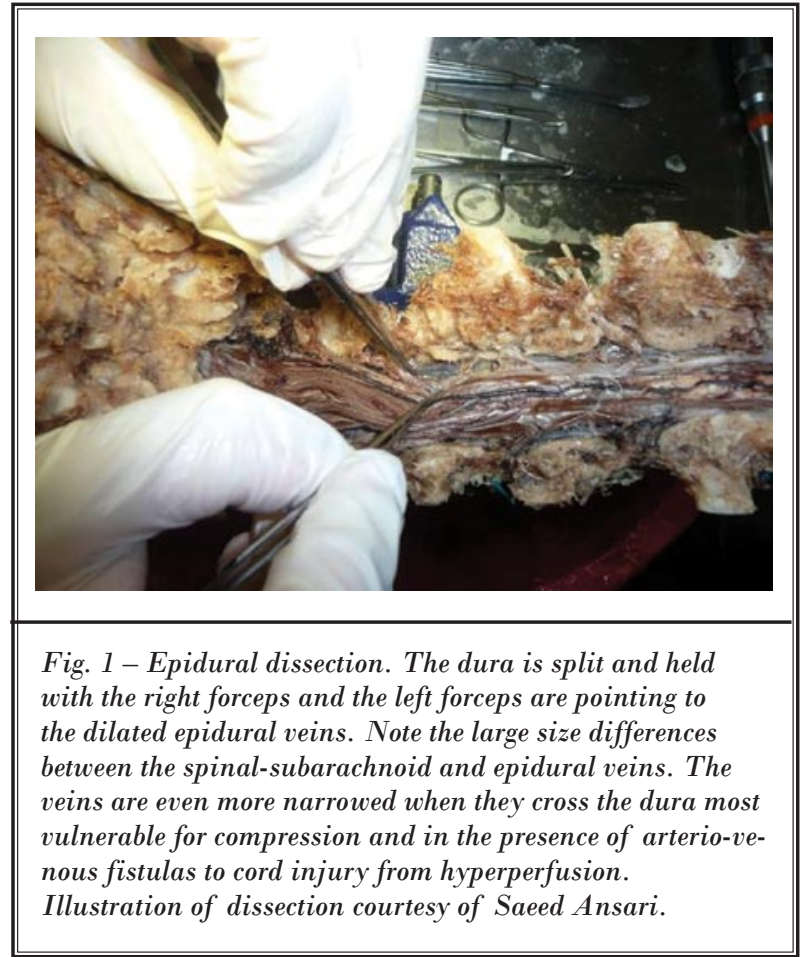

reverse blood flow (19). Evidence for this is based on 3 patients where a routine epidural injection was followed by paralysis secondary to cord injury from hyperperfusion due to venous outflow obstruction which produced a pressure gradient favoring opening of normally present but non functional A-V fistulas. The post injection paralysis was followed by MRO and spinal angiography to diagnose arterio-venous fistulas. These cases responded to subsequent surgical correction with partial recovery (19).

When the pressure builds high enough to contribute to ischemia or pressure insult to neurological structures patients who are conscious with minimal or no sedation may report rapid onset of bilateral upper extremity pain that tends to spread to the chest or back and possibly the lower extremities ("warning signs"). Pain may be followed by ipsi-lateral or bilateral extremity weakness, numbness, and/or paralysis. Patients who are unconscious will be unable to communicate warning signs; however, the above situation may be suspected when after the injection of radioopaque contrast, it does not spread appropriately on the ipsilateral side. Significant concern is warranted when contrast is seen spreading across from the injection site to the contralateral side of the spinal canal without neuroforminal, cephalad, or caudad spread. This pat- 


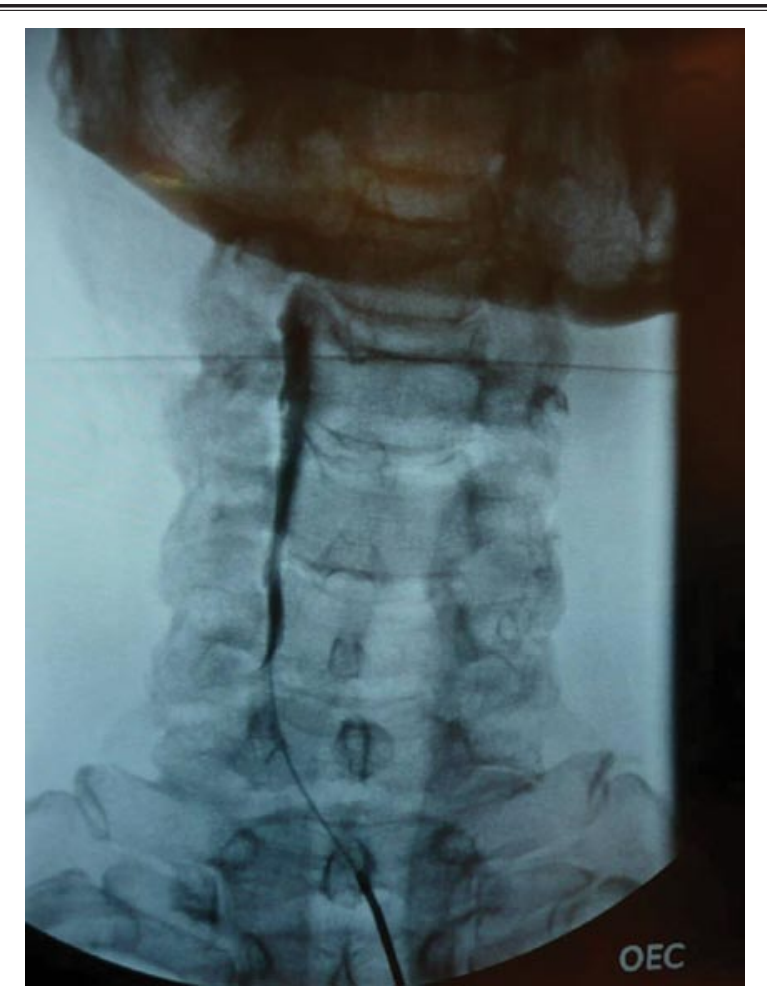

Fig. 2. PVCS

tern has been described as PVCS (peri-venous counter spread), and signifies positive pressure in the lateral epidural space which may lead to bilateral spinal cord compressive effects (Figs. 2-4).

When the pressure of the fluid builds high enough, the fluid can open the peri-venous space outside the veins which transverse the spinal canal from one side to the other, and travel across to the contralateral side, always remaining within the epidural cavity (20). This ipsilateral to contralateral flow of fluid follows a torturous peri-venous path in the epidural cavity corresponding to the course which the veins take across the ventral cervical epidural cavity.

If fluid accumulates and loculation occurs on one or both sides of the spinal cord in the epidural cavity, intense pressure "build-up" in the lateral epidural space may lead to ischemia of the spinal cord. This spinal cord ischemia presents in the conscious patient with the patient complaining of warning signs - the first of these being pain. If the patient is unconscious or not sufficiently responsive as soon as the PVCS contrast spread pattern is recognized, the patient's head should be im-
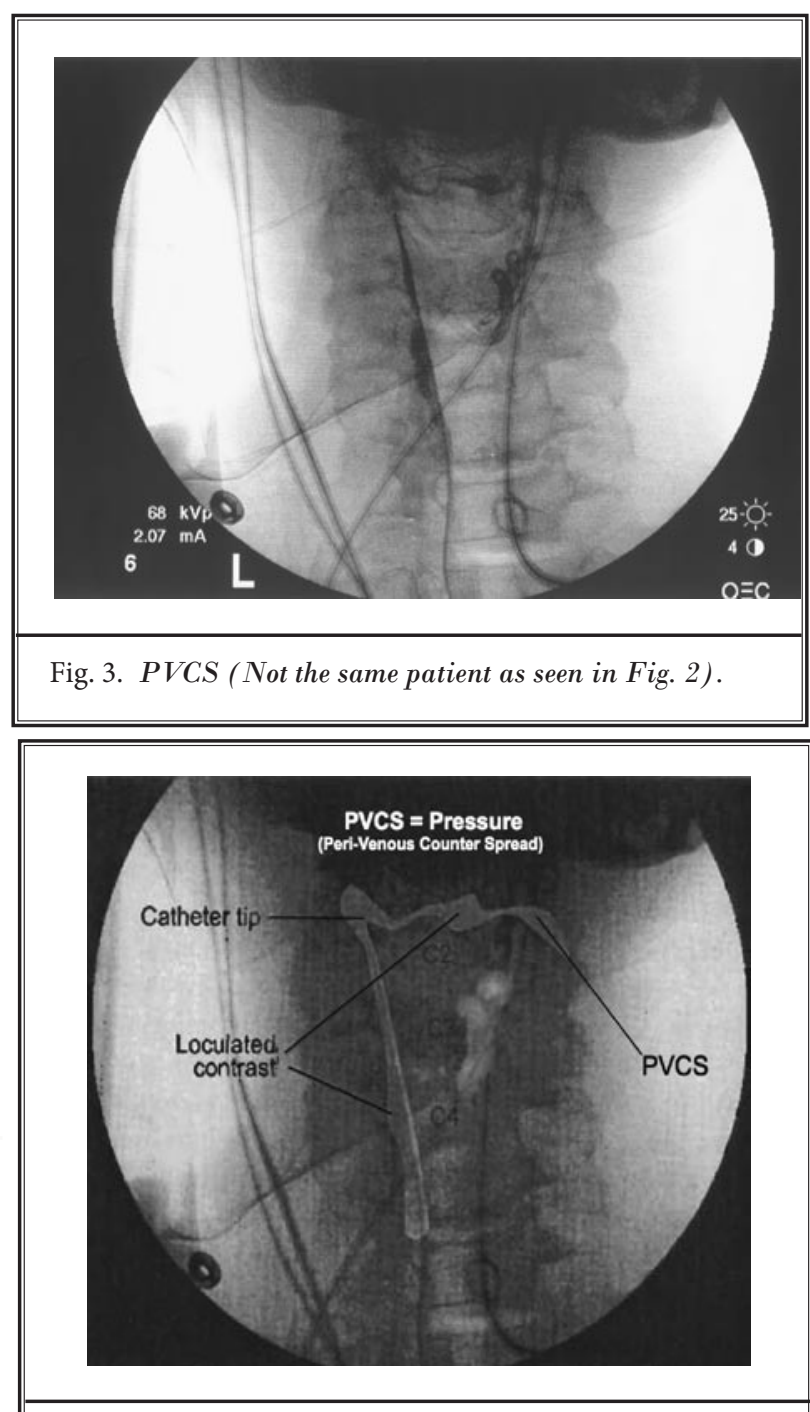

Fig. 2. PVCS (same patient as Fig. 3).

mediately flexed and rotated bringing the chin to the shoulder, flexing and rotating the head continuously from side to side until the symptoms/signs abate (Fig. 5) (20). Movement of the patient's head and neck changes the size of the neuroforamina. It also promotes normal movement of structures within the epidural cavity. The foramina are larger on flexion and smaller on extension (21). Kitagawa and colleagues (21) found that the mean foraminal area in $\mathrm{mm} 2$ for the cervical spine in neutral position was 44 , which increased to 56 with flexion and decreased to 37 with extension. Thus, flexion opened the neural foramen by about $27 \%$, by increasing foraminal height and width, and extension closed the neural 


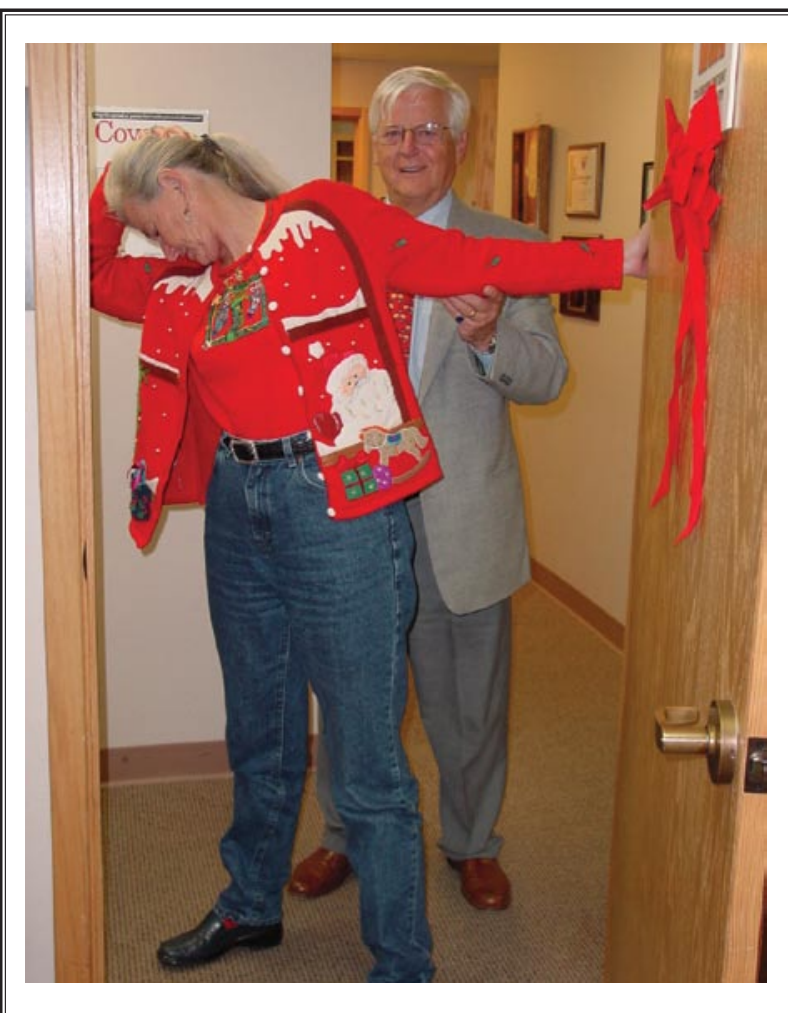

Fig. 5. Flexion and rotation movements.

foramen by $16 \%$, by decreasing the foraminal height and width (21). This increase of mean foraminal area with flexion is augmented roughly $0.5 \%$ over flexion alone, with both flexion and rotation of the head (21). After moving the head as described, fluid may be allowed to escape via lateral runoff through the enlarged neural foramen (Fig. 6) (20). Initial observations related to these issues were originally noted and discussed in 2006 as a potential complication of lysis of epidural adhesions (22), although at that time precise location and structures involved as well as peri-venous counter spread was not fully and completely appreciated.

Prompt recognition of peri-venous counter spread (PVCS) and associated warning signs along with immediate action including flexion and rotation of the patient's head from side to side may facilitate lateral runoff, reduce pressure in the loculated fluid, and potentially avoid catastrophic irreversible neurologic deficits.

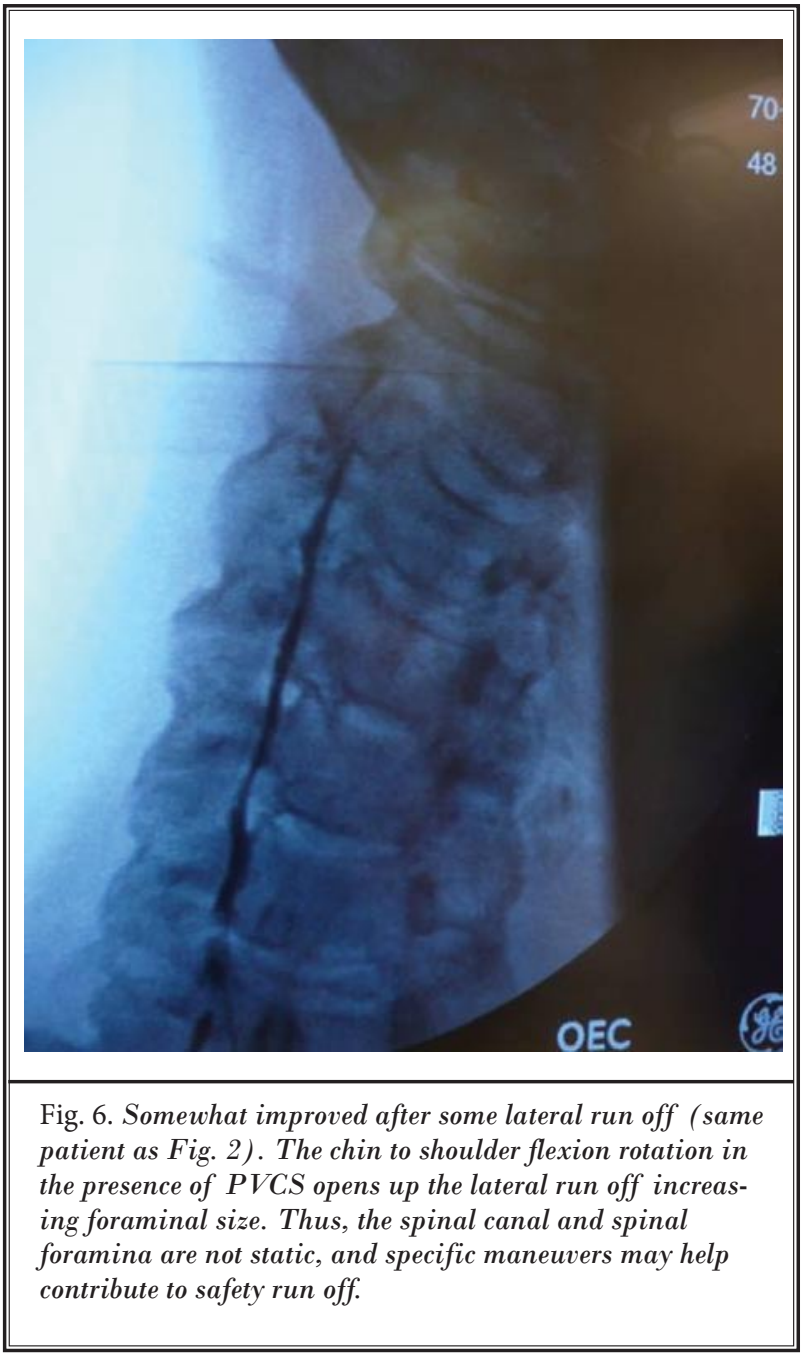

Furthermore, interventionalists should not only be familiar with the signs and symptoms attributable to PVCS - but also should be able to recognize the patterns of contrast spread (Figs. 2-4) - since certain patients may require sedation in order to undergo cervical adhesiolysis procedures. As sedation may mask overt symptoms of PVCS, the quicker the interventionalist can recognize PVCS patterns of contrast spread, the better the chances that maneuvers involving flexion and rotation of the head may lead to a fully reversible and complete recovery. 


\section{References}

1. Turnbull IM, Brieg A, Hassler O. Blood supply of cervical spinal cord in man: A microangiographic cadaver study. J Neurosurg 1966; 24:951-965.

2. Romanes GJ. The arterial blood supply of the human spinal cord. Paraplegia. 1965; 59:199-207.

3. Lazorthes G, Poulhes J, Bastide G, Chancholle AR, Zadeh O. Spinal cord vascularization: Anatomical and physiological study [in French]. Rev Neurol (Paris) 1962; 106:535-557.

4. Lazorthes G, Gouazé A, Bastide G, Santini JJ, Zadeh O, Burdin P. Cervical spinal cord arterial vascularization: study of substitutions anastomoses [in French]. Rev Neurol (Paris) 1966; 115:10551068.

5. Sandson TA, Friedman JH. Spinal cord infarction: Report of 8 cases and review of the literature. Medicine 1989;68: 282-292.

6. Lazorthes G, Gouazé A, Bastide G, Soutoul G, Zadeh O, Santini JJ. La vascularisation artérielle du renflement lombaire. Rev Neurol (Paris) 1966; 114:109122.

7. Zülch KJ, Kurth-Schumacher R. The pathogenesis of "intermittent spinovascular insufficiency" ("spinal claudication of Dejerine") and other vascular syndromes of the spinal cord. Vasc Surg 1970; 4:116-136.

8. Garcin R, Godlewski S, Rondot P. Clinical study of vascular myelopathies [in French]. Rev Neurol (Paris) 1962; 106(6):558-591.

9. Novy J, Carruzzo A, Maeder P, Bogousslavsky J. Spinal cord ischemia: Clinical and imaging patterns, patho- genesis, and outcomes in 27 patients. Arch Neurol 2006; 63:1113-1120.

10. de Haan P, Kalkman CJ, Meylaerts SAG, Lips J, Jacobs MJHM. Development of spinal cord ischemia after clamping of noncritical segmental arteries in the pig. Ann Thorac Surg 1999; 68:12781284.

11. Augoustides J GT. Management of spinal cord perfusion pressure to minimize intermediate-delayed paraplegia: Critical role of central venous pressure. $J$ Thorac Cardiovasc Surg 2008; 136:796.

12. Etz CD, Luehr M, Kari FA, Bodian CA, Smego D, Plestis KA, Griepp RB. Paraplegia after extensive thoracic and thoracoabdominal aortic aneurysm repair: Does critical spinal cord ischemia occur postoperatively? I Thorac Cardiovasc Surg 2008; 135:324-330.

13. Griffiths IR, Pitts LH, Crawford RA, Trench JG. Spinal cord compression and blood flow. I. The effect of raised cerebrospinal fluid pressure on spinal cord blood flow. Neurology 1978; 28:11451451.

14. de Haan P, Kalkman CJ, de Mol BA, Ubags LJ, Veldman DJ, Jacobs MJ. Efficacy of transcranial motor-evoked myogenic potentials to detect spinal cord ischemia during operations for thoracoabdominal aneurysms. J Thorac Cardiovasc Surg 1997; 112:87-101.

15. Shchurova EN, Khudiaev AT. Effect of microcirculation reserves on spinal cord recovery after traumatic injury. Fiziol Cheloveka 2004; 30:54-61.

16. Groen RJ, Groenewegen HJ, Alphen HA, Hoogland PV. Morphology of the hu- man internal vertebral venous plexus: A cadaver study after intravenous Araldite CY 221 injection. Anat Rec 1997; 249:285-294.

17. van der Kuip M, Hoogland P VJM, Groen $R$ JM. Human radicular veins: Regulation of venous reflux in the absense of valves. The Anatomical Record 1999; 254:173-180.

18. Rocco AG, Philip JH, Boas RA, Scott D. Epidural space as a startling resistor and elevation of inflow resistance in a diseased epidural space. Reg Anesth 1997; 22:167-177.

19. Hetts SW, Narvid J, Singh T, Meagher S, Corcoran K, Higashida RT, Dowd CF, Halbach VV. Association between lumbar epidural injection and the development of acute paraparesis in patients with spinal dural arteriovenous fistulas. AJNR Am / Neuroradiol 2007; 28:581-583.

20. Racz GB. Cervical spinal canal loculation and secondary ischemic cord injury - pvcs - perivenous counter spread - danger sign! Pain Prac 2008; 8: 399-403.

21. Kitogawa T, Fujiwara A, Kobayashi N, Saiki K, Tamai K, Saotome K. Morphologic changes in the cervical neural foramen due to flexion and extension (in vitro imaging study). Spine 2004; 29:2821-2825.

22. Racz GB, Heavner JE. Complication associated with lysis of epidural adhesions and epiduroscopy. In: Neal JM, Rathmell JP (eds). Complications of Regional Anesthesia and Pain Medicine. New York, Elsevier, 2006: pp. 301-311. 